\title{
THE DAVIS-GUT LAW FOR INDEPENDENT AND IDENTICALLY DISTRIBUTED BANACH SPACE VALUED RANDOM ELEMENTS
}

\author{
Pingyan Chen, Mingyang Zhang and Andrew Rosalsky
} Jinan Unversity, P. R. China and University of Florida, USA

AbStract. An analog of the Davis-Gut law for a sequence of independent and identically distributed Banach space valued random elements is obtained, which extends the result of $\mathrm{Li}$ and Rosalsky (A supplement to the Davis-Gut law. J. Math. Anal. Appl. 330 (2007), 1488-1493).

\section{INTRODUCTION}

Let $\left\{X, X_{n}, n \geq 1\right\}$ be a sequence of independent and identically distributed random variables. The following theorem, which is related to the classical Hartman-Wintner law of the iterated logarithm (see, Hartman and Wintner, [6]), is well known. As usual we let $\log t=\log _{e} \max \{e, t\}$ for $t \geq 0$.

THEOREM 1.1. The following three statements are equivalent:

$$
\begin{gathered}
E X=0 \text { and } E X^{2}=1, \\
\sum_{n=1}^{\infty} \frac{1}{n} P\left\{\left|\sum_{k=1}^{n} X_{k}\right|>(1+\varepsilon) \sqrt{2 n \log \log n}\right\} \begin{cases}<\infty, & \text { if } \varepsilon>0 \\
=\infty, & \text { if } \varepsilon<0,\end{cases} \\
\sum_{n=1}^{\infty} \frac{\log \log n}{n} P\left\{\left|\sum_{k=1}^{n} X_{k}\right|>(1+\varepsilon) \sqrt{2 n \log \log n}\right\} \begin{cases}<\infty, & \text { if } \varepsilon>0 \\
=\infty, & \text { if } \varepsilon<0 .\end{cases}
\end{gathered}
$$

This result is referred to as the Davis-Gut law. The implication "(1.1) $\Rightarrow(1.2)$ " was formulated by Davis ([3]) with an invalid proof which was corrected by $\mathrm{Li}$ et al. ([11]). The implication "(1.2) $\Rightarrow(1.1)$ " was obtained

2010 Mathematics Subject Classification. 60F15.

Key words and phrases. Davis-Gut law, law of the iterated logarithm, sequence of independent and identically distributed Banach space valued random elements. 
by Gut ([5]). The equivalence between (1.1) and (1.3) was established by Li ([9]). Necessary and sufficient conditions for (1.3) in a Banach space setting were obtained by $\mathrm{Li}([9])$. For moving average processes, the implications "(1.1) $\Rightarrow(1.2) "$ and " $(1.1) \Rightarrow(1.3) "$ were obtained by Chen and Wang $([1])$.

Li and Rosalsky ([10]) provided the following supplement to the DavisGut law. When $h(t) \equiv 1$, it yields the equivalence between (1.1) and (1.2).

THEOREM 1.2. Let $h(\cdot)$ be a positive nondecreasing function on $(0, \infty)$ such that $\int_{1}^{\infty}(t h(t))^{-1} d t=\infty$. Write $\Psi(t)=\int_{1}^{t}(\operatorname{sh}(s))^{-1} d s, t \geq 1$. Then (1.1) and

$$
\sum_{n=1}^{\infty} \frac{1}{n h(n)} P\left\{\left|\sum_{k=1}^{n} X_{k}\right|>(1+\varepsilon) \sqrt{2 n \log \Psi(n)}\right\} \begin{cases}<\infty, & \text { if } \varepsilon>0 \\ =\infty, & \text { if } \varepsilon<0\end{cases}
$$

are equivalent.

Recently, Liu et al. ([12]) extended Theorem 1.2 to moving average processes which then extends the work of Chen and Wang ([1]) by establishing the implication "(1.2) $\Rightarrow(1.1)$ " for moving average processes.

In this paper, we will extend Theorem 1.2 for a sequence of independent and identically distributed Banach space valued random elements.

\section{Preliminaries AND Lemmas}

Let $B$ be a real separable Banach space with norm $\|\cdot\|$ and let $B^{*}$ denote the topological dual space of $B$. We let $B_{1}^{*}$ denote the unit ball of $B^{*}$. Let $(\Omega, \mathcal{F}, P)$ be a probability space. A random element $X$ taking values in $B$ is defined as an $\mathcal{F}$-measurable function from $(\Omega, \mathcal{F})$ into $B$ equipped with the Borel sigma-algebra; we call it a $B$-valued random element for short. The expected value or mean of a $B$-valued random element $X$ is defined to be the Bochner integral and is denoted by $E X$.

LEMMA 2.1. Let $\left\{k_{n}, n \geq 1\right\}$ be a sequence of positive integers and $\left\{X_{n k}, 1 \leq k \leq k_{n}, n \geq 1\right\}$ an array of rowwise independent $B$-valued random elements. Suppose that there exists $\delta>0$ such that $\left\|X_{n k}\right\| \leq \delta$ a.s. for all $1 \leq k \leq k_{n}, n \geq 1$. If $\sum_{k=1}^{k_{n}} X_{n k} \rightarrow 0$ in probability, then $E\left\|\sum_{k=1}^{k_{n}} X_{n k}\right\| \rightarrow 0$ as $n \rightarrow \infty$.

Proof. Let $\left\{X_{n k}^{\prime}, 1 \leq k \leq k_{n}, n \geq 1\right\}$ be an independent copy of $\left\{X_{n k}, 1 \leq k \leq k_{n}, n \geq 1\right\}$. Then by Lemma 2.2 in Chen and Wang ([2]), it suffices to show that

$$
E\left\|\sum_{k=1}^{k_{n}}\left(X_{n k}-X_{n k}^{\prime}\right)\right\| \rightarrow 0 \text { as } n \rightarrow \infty .
$$


It is easy to show that

$$
\sum_{k=1}^{k_{n}}\left(X_{n k}-X_{n k}^{\prime}\right) \rightarrow 0 \text { in probability }
$$

and $\left\|X_{n k}-X_{n k}^{\prime}\right\| \leq 2 \delta$. Therefore by Lemma 2.1 in $\mathrm{Hu}$ et al. ([7]), (2.1) holds and the proof is completed.

Lemma 2.2. Let $0<b_{n} \uparrow \infty$, and $\left\{X, X_{n}, n \geq 1\right\}$ a sequence of independent and identically distributed $B$-valued random elements. If $b_{n}^{-1} \sum_{k=1}^{n} X_{k} \rightarrow$ 0 in probability, then $E\left\|b_{n}^{-1} \sum_{k=1}^{n} X_{k} I\left(\left\|X_{k}\right\| \leq b_{n}\right)\right\| \rightarrow 0$ as $n \rightarrow \infty$.

Then

Proof. Let $\left\{X, X_{n}^{\prime}, n \geq 1\right\}$ be an independent copy of $\left\{X, X_{n}, n \geq 1\right\}$.

$$
b_{n}^{-1} \sum_{k=1}^{n}\left(X_{k}-X_{k}^{\prime}\right) \rightarrow 0 \text { in probability. }
$$

By Lévy's inequality (see display (2.7) in Ledoux and Talagrand [8, p. 47]), for every $t>0$,

$$
P\left\{\max _{1 \leq k \leq n}\left\|X_{k}-X_{k}^{\prime}\right\|>t\right\} \leq 2 P\left\{\left\|\sum_{k=1}^{n}\left(X_{k}-X_{k}^{\prime}\right)\right\|>t\right\},
$$

which by (2.2) ensures that

$$
P\left\{\max _{1 \leq k \leq n}\left\|X_{k}-X_{k}^{\prime}\right\|>b_{n} / 2\right\} \rightarrow 0 \text { as } n \rightarrow \infty .
$$

By Lemma 2.6 of Ledoux and Talagrand [8, p. 51],

$$
\begin{aligned}
n P\left\{\left\|X-X^{\prime}\right\|>b_{n} / 2\right\} & =\sum_{k=1}^{n} P\left\{\left\|X_{k}-X_{k}^{\prime}\right\|>b_{n} / 2\right\} \\
& \leq 2 P\left\{\max _{1 \leq k \leq n}\left\|X_{k}-X_{k}^{\prime}\right\|>b_{n} / 2\right\}
\end{aligned}
$$

when $n$ is sufficiently large. By display (6.1) in Ledoux and Talagrand [8, p. 150],

$$
P\left\{\|X\|>b_{n}\right\} \leq 2 P\left\{\left\|X-X^{\prime}\right\|>b_{n} / 2\right\}
$$

when $n$ is sufficiently large. Therefore by (2.3), (2.4), and (2.5),

$$
n P\left\{\|X\|>b_{n}\right\} \rightarrow 0 \text { as } n \rightarrow \infty .
$$

Note that for any $\varepsilon>0$

$$
P\left\{\left\|\sum_{k=1}^{n} X_{k} I\left(\left\|X_{k}\right\| \leq b_{n}\right)\right\|>\varepsilon b_{n}\right\} \leq n P\left\{\|X\|>b_{n}\right\}+P\left\{\left\|\sum_{k=1}^{n} X_{k}\right\|>\varepsilon b_{n}\right\} .
$$


Then by (2.6) and $b_{n}^{-1} \sum_{k=1}^{n} X_{k} \rightarrow 0$ in probability, it follows that

$$
b_{n}^{-1} \sum_{k=1}^{n} X_{k} I\left(\left\|X_{k}\right\| \leq b_{n}\right) \rightarrow 0
$$

in probability. The conclusion then follows from Lemma 2.1.

The following lemma is due to Einmahl and $\mathrm{Li}([4])$.

Lemma 2.3. Let $Z_{1}, \ldots, Z_{n}$ be independent $B$-valued random elements with mean zero such that for some $s>2, E\left\|Z_{k}\right\|^{s}<\infty, 1 \leq k \leq n$. Then we have for $0<\eta \leq 1, \delta>0$, and $t>0$,

$$
\begin{array}{r}
P\left\{\max _{1 \leq m \leq n}\left\|\sum_{k=1}^{m} Z_{k}\right\| \geq(1+\eta) E\left\|\sum_{k=1}^{n} Z_{k}\right\|+t\right\} \\
\leq \exp \left\{-\frac{t^{2}}{(2+\delta) \Lambda_{n}^{2}}\right\}+C \sum_{k=1}^{n} E\left\|Z_{k}\right\|^{s} / t^{s},
\end{array}
$$

where $\Lambda_{n}^{2}=\sup \left\{\sum_{k=1}^{n} E f^{2}\left(Z_{k}\right): f \in B_{1}^{*}\right\}$ and $C$ is a positive constant depending on $\eta, \delta$ and $s$.

Lemma 2.4. Let $h(t)$ and $\Psi(t)$ be as in Theorem 1.2. Suppose that $X$ is a B-valued random element with

$$
\sum_{n=1}^{\infty} \frac{1}{h(n)} P\{\|X\|>\sqrt{n \log \Psi(n)}\}<\infty .
$$

Then for any $s>2$,

$$
\sum_{n=1}^{\infty} \frac{1}{h(n)} \cdot \frac{1}{(n \log \Psi(n))^{s / 2}} \cdot E\|X\|^{s} I(\|X\| \leq \sqrt{n \log \Psi(n)})<\infty .
$$

Proof. Set $b_{0}=0$ and $b_{n}=\sqrt{n \log \Psi(n)}, n \geq 1$. Note that $\Psi(n) \uparrow$ and therefore $b_{n} / \sqrt{n} \uparrow$. Then $b_{k} / b_{n} \leq \sqrt{k / n}$ whenever $1 \leq k \leq n$. Hence,

$$
\begin{aligned}
& \sum_{n=1}^{\infty} \frac{1}{h(n)} \cdot \frac{1}{(n \log \Psi(n))^{s / 2}} \cdot E\|X\|^{s} I(\|X\| \leq \sqrt{n \log \Psi(n)}) \\
& \quad=\sum_{n=1}^{\infty} \frac{1}{h(n) b_{n}^{s}} \sum_{k=1}^{n} E\|X\|^{s} I\left(b_{k-1}<\|X\| \leq b_{k}\right) \\
& \quad \leq \sum_{n=1}^{\infty} \frac{1}{h(n) b_{n}^{s}} \sum_{k=1}^{n} b_{k}^{s} P\left\{b_{k-1}<\|X\| \leq b_{k}\right\} \\
& \quad=\sum_{k=1}^{\infty} b_{k}^{s} P\left\{b_{k-1}<\|X\| \leq b_{k}\right\} \sum_{n=k}^{\infty} \frac{1}{h(n) b_{n}^{s}}
\end{aligned}
$$




$$
\begin{aligned}
& \leq \sum_{k=1}^{\infty} k^{s / 2} P\left\{b_{k-1}<\|X\| \leq b_{k}\right\} \sum_{n=k}^{\infty} \frac{1}{n^{s / 2} h(n)} \\
& \leq C \sum_{k=1}^{\infty} \frac{k}{h(k)} P\left\{b_{k-1}<\|X\| \leq b_{k}\right\} \\
& \leq \frac{C}{h(1)}+C \sum_{k=1}^{\infty}\left[\frac{k+1}{h(k+1)}-\frac{k}{h(k)}\right] P\left\{\|X\|>b_{k}\right\} \\
& \leq \frac{C}{h(1)}+C \sum_{k=1}^{\infty} \frac{1}{h(k)} P\left\{\|X\|>b_{k}\right\}<\infty,
\end{aligned}
$$

where $C=(s / 2-1)^{-1}$. The proof is completed.

Lemma 2.5. Let $h(n), \Psi(n)$ be as in Theorem 1.2. Then for any B-valued random element $X,(2.7)$ is equivalent to

$$
\sum_{n=1}^{\infty} \frac{1}{h(n)} P\{\|X\|>M \sqrt{n \log \Psi(n)}\}<\infty
$$

for some $M>0$.

Proof. It suffices to prove that (2.7) implies (2.8) for all $0<M<1$. Set $b_{n}=\sqrt{n \log \Psi(n)}, n \geq 1$. Note that $\Psi(n) \uparrow$ and therefore $b_{n} / \sqrt{n} \uparrow$. Then $b_{n} \leq 2^{-1 / 2} b_{2 n}$ for $n \geq 1$. Hence,

$$
\frac{1}{h(2 n)} P\left\{\|X\|>2^{-1 / 2} b_{2 n}\right\} \leq \frac{1}{h(n)} P\left\{\|X\|>b_{n}\right\}
$$

and

$$
\begin{aligned}
\frac{1}{h(2 n+1)} P\left\{\|X\|>2^{-1 / 2} b_{2 n+1}\right\} & \leq \frac{1}{h(2 n)} P\left\{\|X\|>2^{-1 / 2} b_{2 n}\right\} \\
& \leq \frac{1}{h(n)} P\left\{\|X\|>b_{n}\right\}
\end{aligned}
$$

which ensures that

$$
\begin{aligned}
& \sum_{n=1}^{\infty} \frac{1}{h(n)} P\left\{\|X\|>2^{-1 / 2} b_{n}\right\}=\frac{1}{h(1)} P\left\{\|X\|>2^{-1 / 2} b_{1}\right\} \\
& \quad+\sum_{n=1}^{\infty} \frac{1}{h(2 n)} P\left\{\|X\|>2^{-1 / 2} b_{2 n}\right\}+\sum_{n=1}^{\infty} \frac{1}{h(2 n+1)} P\left\{\|X\|>2^{-1 / 2} b_{2 n+1}\right\} \\
& \quad \leq \frac{1}{h(1)} P\left\{\|X\|>2^{-1 / 2} b_{1}\right\}+2 \sum_{n=1}^{\infty} \frac{1}{h(n)} P\{\|X\|>\sqrt{n \log \Psi(n)}\}<\infty
\end{aligned}
$$


Then by mathematical induction, for any integer $k \geq 1$,

$$
\sum_{n=1}^{\infty} \frac{1}{h(n)} P\left\{\|X\|>2^{-k / 2} b_{n}\right\}<\infty .
$$

The proof is completed.

\section{The Main Result and its Proof}

We now state and prove the main result.

Theorem 3.1. Let $h(t)$ and $\Psi(t)$ be as in Theorem 1.2. Let $\left\{X, X_{n}, n \geq\right.$ $1\}$ be a sequence of independent and identically distributed $B$-valued random elements. Suppose that

$$
(\sqrt{n \log \Psi(n)})^{-1} \sum_{k=1}^{n} X_{k} \rightarrow 0 \text { in probability. }
$$

(i) Suppose that (2.7) holds and

$$
E X=0, E f^{2}(X)<\infty \quad \forall f \in B^{*} .
$$

Then

$$
\begin{aligned}
& \sum_{n=1}^{\infty} \frac{1}{n h(n)} P\left\{\left\|\sum_{k=1}^{n} X_{k}\right\|>(1+\varepsilon) \sqrt{2 \sigma^{2} n \log \Psi(n)}\right\} \begin{cases}<\infty, & \text { if } \varepsilon>0 \\
=\infty, & \text { if } \varepsilon<0,\end{cases} \\
& \text { where } \sigma^{2}=\sup \left\{E f^{2}(X): f \in B_{1}^{*}\right\}
\end{aligned}
$$

(ii) Conversely, suppose that

$$
\sum_{n=1}^{\infty} \frac{1}{n h(n)} P\left\{\left\|\sum_{k=1}^{n} X_{k}\right\|>M \sqrt{n \log \Psi(n)}\right\}<\infty
$$

holds for some $M>0$. Then (2.7) and (3.1) hold.

Proof. Set $a_{n}=\sqrt{2 \sigma^{2} n \log \Psi(n)}, b_{n}=\sqrt{n \log \Psi(n)}, n \geq 1$ and

$$
X_{n k}=X_{k} I\left(\left\|X_{k}\right\| \leq b_{n}\right), Z_{n k}=X_{n k}-E X_{n k}, 1 \leq k \leq n, n \geq 1 .
$$

(i) Suppose that (2.7) and (3.1) hold. We first prove that

$$
\sum_{n=1}^{\infty} \frac{1}{n h(n)} P\left\{\left\|\sum_{k=1}^{n} X_{k}\right\|>(1+\varepsilon) a_{n}\right\}<\infty \forall \varepsilon>0 .
$$

Note that for any $\varepsilon>0$,

$$
P\left\{\left\|\sum_{k=1}^{n} X_{k}\right\|>(1+\varepsilon) a_{n}\right\} \leq n P\left\{\|X\|>b_{n}\right\}+P\left\{\left\|\sum_{k=1}^{n} X_{n k}\right\|>(1+\varepsilon) a_{n}\right\} .
$$


Hence, by (2.7), to prove (3.4), it suffices to prove that

$$
\sum_{n=1}^{\infty} \frac{1}{n h(n)} P\left\{\left\|\sum_{k=1}^{n} X_{n k}\right\|>(1+\varepsilon) a_{n}\right\}<\infty \forall \varepsilon>0 .
$$

By Lemma 2.2,

$$
\frac{1}{b_{n}}\left\|\sum_{k=1}^{n} E X_{n k}\right\| \leq \frac{1}{b_{n}} E\left\|\sum_{k=1}^{n} X_{n k}\right\| \rightarrow 0 \text { as } n \rightarrow \infty
$$

and

$$
\frac{1}{b_{n}} E\left\|\sum_{k=1}^{n} Z_{n k}\right\| \leq \frac{2}{b_{n}} E\left\|\sum_{k=1}^{n} X_{n k}\right\| \rightarrow 0 \text { as } n \rightarrow \infty .
$$

Then to prove (3.5), it suffices to prove that

(3.6) $\sum_{n=1}^{\infty} \frac{1}{n h(n)} P\left\{\left\|\sum_{k=1}^{n} Z_{n k}\right\|>2 E\left\|\sum_{k=1}^{n} Z_{n k}\right\|+(1+\varepsilon) a_{n}\right\}<\infty \forall \varepsilon>0$.

By Lemma 2.3, for some $s>2$ and any $\delta>0$

$$
\begin{gathered}
P\left\{\left\|\sum_{k=1}^{n} Z_{n k}\right\|>2 E\left\|\sum_{k=1}^{n} Z_{n k}\right\|+(1+\varepsilon) a_{n}\right\} \\
\leq \exp \left\{-\frac{(1+\varepsilon)^{2} a_{n}^{2}}{(2+\delta) \Lambda_{n}^{2}}\right\}+\frac{C}{b_{n}^{s}} \sum_{k=1}^{n} E\left\|Z_{n k}\right\|^{s},
\end{gathered}
$$

where $\Lambda_{n}^{2}=\sup \left\{\sum_{k=1}^{n} E f^{2}\left(Z_{n k}\right): f \in B_{1}^{*}\right\}$. Note that for all $f \in B_{1}^{*}$,

$$
\begin{aligned}
E f^{2}\left(Z_{n k}\right) & =E f^{2}\left(X_{n k}\right)-\left(E f\left(X_{n k}\right)\right)^{2} \leq E f^{2}\left(X_{n k}\right) \\
& \leq E f^{2}(X), \quad 1 \leq k \leq n, n \geq 1 .
\end{aligned}
$$

Therefore $\Lambda_{n}^{2} \leq n \sigma^{2}, n \geq 1$. Choose $\delta>0$ small enough so that $t=2(1+$ $\varepsilon)^{2} /(2+\delta)>1$. Then

$$
\begin{aligned}
\sum_{n=1}^{\infty} \frac{1}{n h(n)} \exp \left\{-\frac{(1+\varepsilon)^{2} a_{n}^{2}}{(2+\delta) \Lambda_{n}}\right\} & \leq \sum_{n=1}^{\infty} \frac{1}{n h(n)} \exp \left\{-\frac{(1+\varepsilon)^{2} a_{n}^{2}}{(2+\delta) \Lambda_{n}}\right\} \\
& \leq \sum_{n=1}^{\infty} \frac{1}{n h(n)} \exp \{-t \log \Psi(n)\} \\
& \leq \sum_{n=1}^{\infty} \frac{1}{n h(n)} \cdot \frac{1}{(\Psi(n))^{t}}<\infty
\end{aligned}
$$


since $\int_{1}^{\infty} d x /\left[x h(x) \Psi^{t}(x)\right]<\infty$. By the $C_{r}$-inequality, Hölder's inequality, and Lemma 2.4,

$$
\begin{aligned}
& \sum_{n=1}^{\infty} \frac{1}{n h(n)} \cdot \frac{1}{b_{n}^{s}} \sum_{k=1}^{n} E\left\|Z_{n k}\right\|^{s} \\
& \quad \leq \sum_{n=1}^{\infty} \frac{1}{h(n)} \cdot \frac{1}{(n \log \Psi(n))^{s / 2}} \cdot E\|X\|^{s} I(\|X\| \leq \sqrt{n \log \Psi(n)})<\infty
\end{aligned}
$$

By (3.7), (3.8), and (3.9), (3.6) holds and hence (3.4) holds as was argued above.

Now we prove that

$$
\sum_{n=1}^{\infty} \frac{1}{n h(n)} P\left\{\left\|\sum_{k=1}^{n} X_{k}\right\|>(1+\varepsilon) a_{n}\right\}=\infty \forall \varepsilon<0 .
$$

For any $f \in B^{*}$, by (3.1), $E f(X)=0$ and $E f^{2}(X)<\infty$. Then by the implication " $(1.1) \Rightarrow(1.4)$ " in Theorem 1.2, for all $\varepsilon<0$

$$
\sum_{n=1}^{\infty} \frac{1}{n h(n)} P\left\{\left|\sum_{k=1}^{n} f\left(X_{k}\right)\right|>(1+\varepsilon) \sqrt{2 E f^{2}(X) n \log \Psi(n)}\right\}=\infty
$$

Note that for any $f \in B_{1}^{*},\left|\sum_{k=1}^{n} f\left(X_{k}\right)\right| \leq\left\|\sum_{k=1}^{n} X_{k}\right\|$ and so it follows from (3.11) that for all $f \in B_{1}^{*}$, for all $\varepsilon<0$

$$
\sum_{n=1}^{\infty} \frac{1}{n h(n)} P\left\{\left\|\sum_{k=1}^{n} X_{k}\right\|>(1+\varepsilon) \sqrt{2 E f^{2}(X) n \log \Psi(n)}\right\}=\infty .
$$

Hence (3.10) holds by (3.12) and $\sigma^{2}=\sup \left\{E f^{2}(X): f \in B_{1}^{*}\right\}$. Combining (3.4) and (3.10) yields (3.2).

(ii) Assume that (3.3) holds for some $M>0$. Then for any $f \in B_{1}^{*}$,

$$
\sum_{n=1}^{\infty} \frac{1}{n h(n)} P\left\{\left|\sum_{k=1}^{n} f\left(X_{k}\right)\right|>M b_{n}\right\}<\infty .
$$

Then by the implication " $(2.3) \Rightarrow(2.4)$ " of Li and Rosalsky $([10])$, it follows that $E f(X)=0$ and $E f^{2}(X)<\infty$. Hence (3.1) holds.

Let $\left\{X^{\prime}, X_{n}^{\prime}, n \geq 1\right\}$ be an independent copy of $\left\{X, X_{n}, n \geq 1\right\}$. Then by the same argument as in the proof of Lemma 2.2,

$$
\begin{aligned}
n P\left\{\|X\|>4 M b_{n}\right\} & \leq 8 P\left\{\left\|\sum_{k=1}^{n}\left(X_{k}-X_{k}^{\prime}\right)\right\|>2 M b_{n}\right\} \\
& \leq 16 P\left\{\left\|\sum_{k=1}^{n} X_{k}\right\|>M b_{n}\right\},
\end{aligned}
$$


which by (3.3) ensures that

$$
\sum_{n=1}^{\infty} \frac{1}{h(n)} P\left\{\|X\|>4 M b_{n}\right\}<\infty
$$

and so (2.7) holds by Lemma 2.5. The proof is completed.

REMARK 3.2. A sufficient condition for (2.7) is $E\|X\|^{2}<\infty$. Indeed,

$$
\begin{aligned}
\sum_{n=1}^{\infty} \frac{1}{h(n)} P\{\|X\|>\sqrt{n \log \Psi(n)}\} & \leq \frac{1}{h(1)} \sum_{n=1}^{\infty} P\{\|X\|>\sqrt{n}\} \\
& \leq \frac{1}{h(1)} E\|X\|^{2}<\infty .
\end{aligned}
$$

REMARK 3.3. Some examples of moment conditions which are equivalent to (2.7) for various choices of $h(\cdot)$ will now be given.

CASE (i). Set $h(t)=(\log \log t)^{b}$ where $b \geq 0$. Then $\log \Psi(t) \sim \log \log t$ as $t \rightarrow \infty$ and (2.7) is equivalent to $E\|X\|^{2} /(\log \log \|X\|)^{b+1}<\infty$.

CASE (ii). Set $h(t)=(\log t)^{r}$ where $0 \leq r<1$. Then $\log \Psi(t) \sim(r-$ 1) $\log \log t$ as $t \rightarrow \infty$ and (2.7) is equivalent to $E\|X\|^{2} /\left[(\log \|X\|)^{r} \log \log \|X\|\right]$ $<\infty$.

CaSE (iii). Set $h(t)=\log t$. Then $\log \Psi(t) \sim \log \log \log t$ as $t \rightarrow \infty$ and (2.7) is equivalent to $E\|X\|^{2} /[(\log \|X\|) \log \log \log \|X\|]<\infty$.

CASE (iv). In Case (i), take $b=0$, or in Case (ii), take $r=0$. Then (2.7) is equivalent to $E\|X\|^{2} / \log \log \|X\|<\infty$.

ACKNOWLEDGEMENTs.

The research of Chen is supported by the National Natural Science Foundation of China (No. 71471075).

\section{REFERENCES}

[1] P.Y. Chen and D.C. Wang, Convergence rates for probabilities of moderate deviations for moving average processes, Acta Math. Sin. (Engl. Ser.) 24 (2008), 611-622.

[2] P.Y. Chen and D.C. Wang, $L^{r}$ convergence for B-valued random elements, Acta Math. Sin. (Engl. Ser.) 28 (2012), 857-868.

[3] J.A. Davis, Convergence rates for the law of the iterated logarithm, Ann. Math. Statist. 39 (1968), 1479-1485.

[4] U. Einmahl and D. Li, Characterization of LIL behavior in Banach space, Trans. Amer. Math. Soc. 360 (2008), 6677-6693.

[5] A. Gut, Convergence rates for probabilities of moderate deviations for sums of random variables with multidimensional indices, Ann. Probab. 8 (1980), 298-313.

[6] P. Hartman and A. Wintner, On the law of the iterated logarithm, Amer. J. Math. 63 (1941), 169-176.

[7] T.-C. Hu, A. Rosalsky, D. Szynal and A.I. Volodin, On complete convergence for arrays of rowwise independent random elements in Banach spaces, Stochastic Anal. Appl. 17 (1999), 963-992.

[8] M. Ledoux and M. Talagrand, Probability in Banach spaces. Isoperimetry and processes, Springer-Verlag, Berlin, 1991. 
[9] D.L. Li, Convergence rates of law of iterated logarithm for B-valued random variables, Sci. China Ser. A 34 (1991), 395-404.

[10] D. Li and A. Rosalsky, A supplement to the Davis-Gut law, J. Math. Anal. Appl. 330 (2007), 1488-1493.

[11] D.L. Li, X. C. Wang and M.B. Rao, Some results on convergence rates for probabilities of moderate deviations for sums of random variables, Internat. J. Math. Math. Sci. 15 (1992), 481-497.

[12] X. Liu, H. Qian and L. Cao, The Davis-Gut law for moving average processes, Statist. Probab. Lett. 104 (2015), 1-6.

P. Chen

Department of Mathematics

Jinan Unversity

Guangzhou, 510630

P. R. China

E-mail: tchenpy@jnu.edu.cn

M. Zhang

Department of Mathematics

Jinan University

Guangzhou, 510630

P. R. China

E-mail: zmy1021@qq.com

A. Rosalsky

Department of Statistics

University of Florida

Gainesville, FL 32611

USA

E-mail: rosalsky@stat.ufl.edu

Received: $20 \cdot 6 \cdot 2016$. 